\title{
Index System Reduction Method Based on the Index Similarity
}

\author{
Ai WANG, Xuedong GAO
}

\begin{abstract}
Multi-attribute decision making (MADM) always suffers from the result inconsistency and computational complexity problem, due to numbers of redundant and relational attributes (indexes) of the initial evaluation index system. Therefore, this paper studies the index system (IS) reduction problem through selecting the most representative indicator from each index subsystem after the IS structure partition. First, we propose and demonstrate the Index Subsystem Judgement theorem to improve the efficiency of the classic system structure partition algorithm. Second, an algorithm of index system reduction based on the index similarity (ISRS) is put forward. The ISRS is able to reduce the index quantity while still keeping the index meaning. Third, we define the direction loss rate to measure the evaluation ability loss of the IS during reduction. The algorithm is tested for a synthetic dataset to compare the proposed ISRS with different index reduction algorithms, followed by an extensive experimentation with a real-world financial dataset. Experiment results illustrate that our proposed method is able to obtain more accessible and available reduction results in practice.
\end{abstract}

Keywords: direction loss rate; Index Subsystem Judgment theorem; index system reduction; index similarity

\section{INTRODUCTION}

Multi-attribute decision making (MADM) is a subfield of operations research, concerned with selecting the best alternative through the evaluation of the whole set of attributes which are hard to quantify, incommensurable or incomparable $[2,35]$. A number of redundant or relational attributes (indexes) might increase the potential internal inconsistency and computational complexity of the MADM methods, such as analytic hierarchy process (AHP) $[37,39]$. To deal with this drawback, an appropriate index reduction should be implemented.

Since an index system is exactly a system which consists of different indexes (elements) with specific structure (relation), the index system reduction problem could be definitely transformed to the system structure partition problem, that is selecting the most representative index from each index subsystem.

Formally, let $S$ denote the initial index system, the task of index system reduction is to partition $S$ into several index subsystems $S_{i}$ and utilize one index $X_{i k}$ to replace each subsystem, where (1) $S_{i} \neq \emptyset$, (2) $\cup S_{i}=S$, (3) $S_{i} \cap$ $S_{j}=\emptyset$. Here, $X_{i k} \in S_{i} \subseteq S$ is an index and the final reduced index system $O \subseteq S$.

Tab.1 is a common evaluation index system on students' learning performance, including every course score as well as the total and average grade. It can be seen that not only there is the direct linear relation between the total and average grade (that is if one of the Total and Average is known, the other could be calculated through simply multiplying a coefficient), also the performance of different students on the same course shares some similarity, such as Math and Physics.

Obviously, in order to improve the evaluation efficiency of the index system in Tab.1, it is better to just remove one index from each subsystem (i.e., \{Math, Physics\} and \{Total, Average\}), which successfully reduces the index quantity while still keeping the index meaning. We study the feature of these two subsystems and find out that indexes in the same subsystem are much more similar to each other than to those in other subsystems, which illustrates that the partition principle of index subsystems is on the basis of the index similarity.
Therefore, this paper focuses on the index system reduction problem based on the index similarity. The main contributions are as follows. First, we propose and demonstrate the Index Subsystem Judgement theorem and its inference, which can improve the efficiency of the system structure partition process, even be valid for other problems (e.g. high dimensional data pre-processing, knowledge discovery). Second, an algorithm of index system reduction based on the index similarity (ISRS) is also proposed. The ISRS is able to reduce the index quantity while still keeping the index meaning compared to traditional index reduction algorithms, which shows great advantages in practice. Third, we define the direction loss rate to measure the evaluation ability loss of the index system, which could verify and evaluate the results obtained by the ISRS.

The rest of the paper is organized as follows. Section 2 presents the previous works related to this research. Section 3 presents the methodology of the index system reduction, including the theorem proof and algorithm. Section 4.1 conducts several comparison experiments related to different index system reduction algorithms and similarity evaluation indices on a synthetic dataset. Besides, experiments in Section 4.2 further verify the effectiveness and stability of the proposed method on a real financial data set. The paper is concluded in Section 5.

Table 1 An index system on students' learning performance

\begin{tabular}{|l|c|c|c|c|c|c|}
\hline Student & English & Chinese & Math & Physics & Total & Average \\
\hline Tom & 98 & 90 & 92 & 91 & 371 & 92.75 \\
\hline Sherry & 96 & 74 & 87 & 88 & 345 & 86.25 \\
\hline Bill & 57 & 87 & 45 & 49 & 238 & 59.50 \\
\hline Jack & 84 & 79 & 60 & 60 & 283 & 70.75 \\
\hline Mary & 47 & 92 & 61 & 59 & 259 & 64.75 \\
\hline
\end{tabular}

\section{LITERATURE REVIEW}

\subsection{Index System Reduction Algorithms}

This section reviews the conventional index system reduction algorithms, such as the principal component analysis (PCA) [4], rough set (RS) theory [12] and independent component analysis (ICA) [6].

Tab. 2 describes the comparison of the PCA, RS theory and the proposed algorithm ISRS (see Section 3). Although all three algorithms are able to solve the reduction problem, 
the emphasis is somewhat different $[8,15]$. For example, the RS theory mainly focuses on the attribute reduction problem $[23,28]$ and shows much potential in the multilabel learning $[29,36]$, while the PCA is usually applied to the dimension reduction problem in the machine learning $[9,16]$ and multi-objective optimization (MOO) $[18,20]$. What's more, the RS theory reduces attributes based on the partition of equivalence relation, which can be accomplished through the evaluation metric [30, 31], like mutual information and information entropy [10, 38]. In general, the RS theory achieves better performance on the categorical attribute dataset $[1,11]$. The PCA reduces dimensions based on the variance additivity of irrelevant principal components $[21,22]$, which can be evaluated by the contribution rate $[19,27]$. The purpose of the ICA is similar to the PCA, which aims to obtain independent components for dimension reduction $[25,26]$. However, the proposed ISRS in this paper achieves the index reduction based on the index similarity, especially for the multi-attribute decision making (MADM). Also, direction loss rate is put forward, in order to measure the evaluation ability loss of reduced index system.

Besides, the results of three algorithms are quite different, that is the decision table (of RS theory), new principal components (of PCA) and index system (of ISRS) $[3,33,34]$. From the perspective of ISRS, both independent components (of ICA) and principal components are the combination of initial indexes, which means compared to the ISRS, the PCA and ICA have the same effect. Since this paper aims to study the index system reduction problem of the numerical dataset, the comparison experiments of different algorithms in Section 4.1 are mainly conducted between the PCA and ISRS.

Table 2 The comparison of index system reduction algorithms

\begin{tabular}{|l|l|l|l|}
\hline \multicolumn{1}{|c|}{ Method } & \multicolumn{1}{|c|}{ RS theory } & \multicolumn{1}{c|}{ PCA } & Proposed ISRS \\
\hline Purpose & Attribute reduction & Dimension reduction & Index similarity \\
\hline Principle & Equivalence relation & Variance additivity & Direction loss rate \\
\hline Evaluation Metric & Mutual information & Contribution rate & Index system \\
\hline Result & Decision table & Principal components & Multi-attribute decision making(MADM) \\
\hline Typical Field & Multi-label learning & Multi-objective optimization (MOO) & \\
\hline
\end{tabular}

\subsection{Similarity Evaluation Indices}

Similarity evaluation indices is a popular research field of data mining, and are wildly utilized in clustering and classification algorithms [5]. We review three typical metrics as follows.

Give an index system $S=\left\{X_{1}, X_{2}, \ldots, X_{n}\right\}$, let $\overrightarrow{x_{k}}=$ $\left(v_{k 1}, v_{k 2}, \ldots, v_{k m}\right)$ represents the sample vector of index $X_{k}$, and $\overrightarrow{x_{l}}=\left(v_{l 1}, v_{l 2}, \ldots, v_{l m}\right)$ represents the sample vector of index $X_{l}$. The Cosine distance $d$, Euclidean distance $\dot{d}$, Mahalanobis distance $\ddot{d}$ of index $X_{k}$ and $X_{l}$ is:

$$
\begin{aligned}
& d\left(X_{k}, X_{l}\right)=1-\overrightarrow{x_{k}} \cdot \overrightarrow{x_{l}} /\left(\left|\overrightarrow{x_{k}}\right|\left|\overrightarrow{x_{l}}\right|\right) \\
& \dot{d}\left(X_{k}, X_{l}\right)=\sqrt{\sum_{t=1}^{m}\left(v_{k t}-v_{l t}\right)^{2}} \\
& \ddot{d}\left(X_{k}, X_{l}\right)=\sqrt{\left(\overrightarrow{x_{k}}-\overrightarrow{x_{l}}\right)^{T} \sum^{-1}\left(\overrightarrow{x_{k}}-\overrightarrow{x_{l}}\right)}
\end{aligned}
$$

where $\sum$ is the covariance matrix of index system $S$.

Cosine distance measures the direction difference of index vectors, Euclidean distance measures the length difference of index vectors, and Mahalanobis distance measures the covariance distance of index vectors. Although Mahalanobis distance overcomes the correlation between indicators and is scale-invariant, it strictly requires the number of indexes must be larger than the number of samples (because we want to calculate the similarity of different indexes not samples), which is not consistent with the index dataset in practice. Thus, the comparison experiments of different indices in Section 4.1 are mainly conducted between the Cosine distance and Euclidean distance.

\section{INDEX SYSTEM REDUCTION METHOD BASED ON THE DIRECTION LOSS RATE}

This section studies the theoretical framework and algorithm of the index system reduction problem. Since the index system reduction is exactly the system structure partition in essence, the classic method of the system structure partition, that is the interpretative structural modeling (ISM), is applied to our research [14, 32].

Fig. 1 shows the principle of the index system reduction method based on the index similarity. There are four major phases. First, calculate the index similarity through sufficient samples, in order to identify the relation between different indicators. Then, divide the initial index system into several index subsystems by means of the improved ISM. After that, replace each subsystem with its most representative index, and obtain the reduced index system. Finally, calculate the evaluation ability loss during reduction via the proposed metric, direction loss rate.

According to the example of students' learning performance in Section 1, since the indexes with linear relation ought to be reduced (like Total and Average), the direction difference of different indexes plays a more significant role than the length difference. Thus, we take the Cosine distance as the similarity evaluation indices (see Eq. (1)).

Refer to the ISM, the final similarity of different elements depends on the overall relation, not only limited to the direct relation (i.e., Cosine distance) [7]. Consequently, we judge the relationship between indexes through the overall relation in the reachable matrix. Given index $X_{k}$ and $X_{l}$, the judgement function of the overall cosine distance $\bar{d}\left(X_{k}, X_{l}\right)$ is:

$J\left(X_{k}, X_{l}\right)= \begin{cases}1, & \bar{d}\left(X_{k}, X_{l}\right) \leq \delta \\ 0, & \bar{d}\left(X_{k}, X_{l}\right)>\delta\end{cases}$

Where $\delta$ represents the similarity threshold, and $J\left(X_{k}, X_{l}\right)=1$ means $X_{k}$ is definitely similar to $X_{l}$, that is $X_{k}$ can reach $X_{l}$; otherwise, $J\left(X_{k}, X_{l}\right)=0$ means $X_{k}$ is not similar to $X_{l}$, that is $X_{k}$ cannot reach $X_{l}$. 

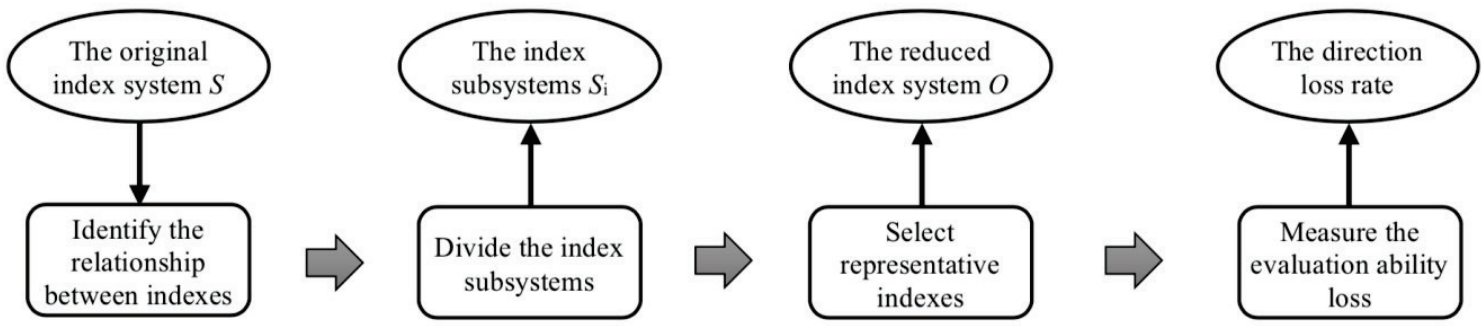

Figure 1 The principle of the index system reduction method based on the index similarity

Theorem 1 (Index Subsystem Judgment theorem). In a reachable matrix, all the indexes with the same reachable set or the same antecedent set only belong to one index subsystem.

Proof. Given an index system $S$ and index $X_{k}, X_{l} \in S$.

Assume that $X_{k}, X_{l}$ have the same reachable set, that is $A\left(X_{k}\right)=A\left(X_{l}\right)$.

Because $X_{k} \in A\left(X_{k}\right)$ and $A\left(X_{k}\right)=A\left(X_{l}\right), X_{k} \in$ $A\left(X_{l}\right)$. Consequently, the index $X_{l}$ can reach $X_{k}$. In the same way, the index $X_{k}$ can reach $X_{l}$.

That $X_{k}, X_{l}$ reach each other means they belong to one strong connected domain (subsystem).

Hence, all the indexes with the same reachable set belong to one subsystem.

If index $X_{l}$ also belongs to another subsystem that contains the index $X_{p}$, while $X_{k}$ does not, then is $X_{p} \in$ $A\left(X_{l}\right)$ and $X_{p} \notin A\left(X_{k}\right)$.

However, $X_{p} \in A\left(X_{l}\right)$ and $X_{p} \notin A\left(X_{k}\right)$ contradicts the assumption $A\left(X_{k}\right)=A\left(X_{l}\right)$.

Thus, all the indexes with the same reachable set only belong to one subsystem.

The antecedent set can be used to prove the same conclusion.

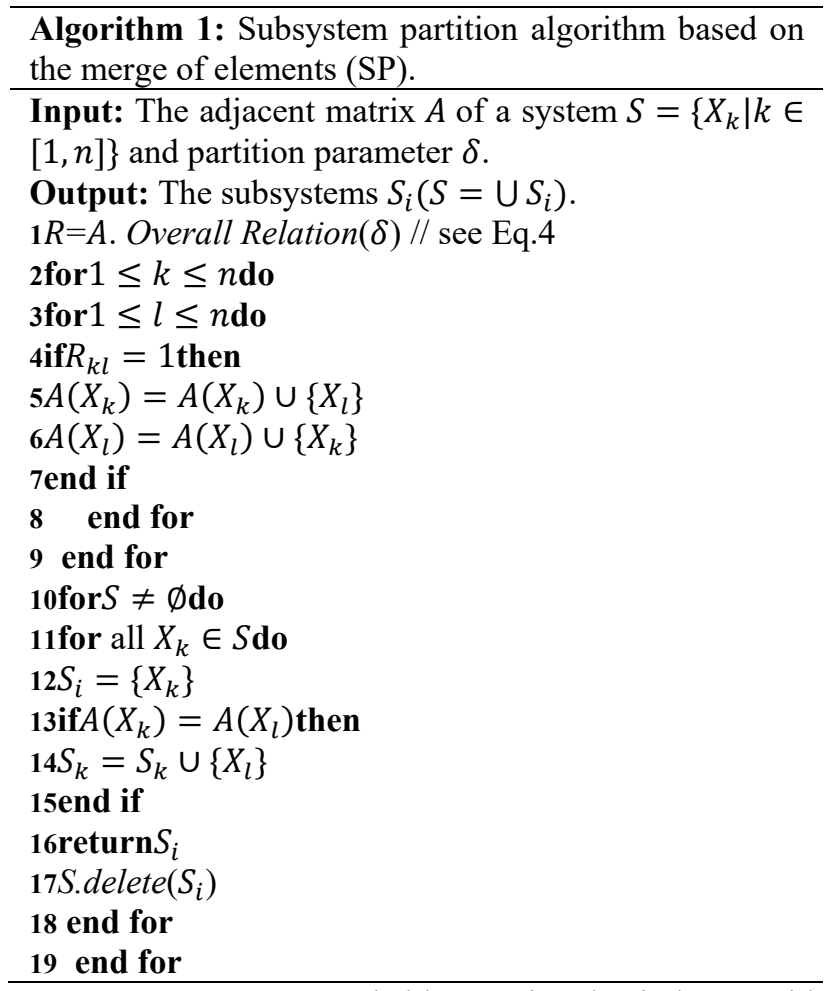

Inference. In a reachable matrix, the indexes with different reachable sets are bound to have different antecedent sets, vice versa.
We improve the ISM via the Theorem 1, and the pseudo code is shown in Algorithm 1. The time complexity of the SP is $O\left(n^{2} / 2\right)$, where $\mathrm{n}$ is the total number of indexes, which obtains an obvious improvement towards the classic algorithm $O\left(n^{3}\right)$.

Phase 3 (that is select a representative index) is the core index reduction process after the preparation Phase 1 and 2, which share the same essence to the attribute selection problem in data mining research field [5]. Hence, we take the wildly-used attribute selection metric, information entropy, as the measurement of index evaluation ability during reduction (see Eq.5) [24].

Given the index vector $\overrightarrow{x_{k}}=\left(v_{k t}\right),(t \in[1, m])$ of index $X_{k}, v_{k t}$ represents the sample value (of sample $O_{t}$ on index $\left.X_{k}\right)$, and $p\left(v_{k t}\right)$ represents the probability of value $v_{k t}$. The evaluation ability of index $X_{k}$ is:

$H\left(X_{k}\right)=-\sum_{t=1}^{m} p\left(v_{k t}\right) \log \left(p\left(v_{k t}\right)\right)$

where $0<p\left(v_{k t}\right) \leq 1$, and $\sum_{t=1}^{m} p\left(v_{k t}\right)=1$.

As for the entropy decrease effect, the index with larger information entropy should be abandoned due to the poor information value; while the index with smaller information entropy should be reserved due to the rich information value [17].

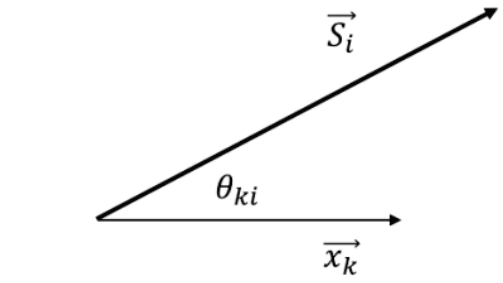

Figure 2 The index replacement process during reduction

Moreover, it is also necessary to measure the evaluation ability loss of the index system after reduction. Fig. 2 shows the index replacement process on one index subsystem. Let $\overrightarrow{x_{k}}$ represent the vector of index $X_{k}$ in the index subsystem $S_{i}$, and $\overrightarrow{S_{l}}$ represent the resultant vector of subsystem $S_{i}$, that is $\overrightarrow{S_{l}}$ consists of all the index vectors in $S_{i}$. If using index $X_{k}$ to replace the whole subsystem $S_{i}$, the evaluation direction of the index subsystem changes from $\overrightarrow{S_{l}}$ to $\overrightarrow{x_{k}}$. Thus, the evaluation ability loss of $S_{i}$ during reduction is exactly the evaluation direction $\theta_{k i}$.

Definition 1 (Subsystem Direction Loss Rate). Let $S_{i}=\left\{X_{k} \mid k \in[1, u]\right\}$ represent an index subsystem, $\overrightarrow{x_{k}}=\left(v_{k 1}, v_{k 2}, \ldots, v_{k m}\right)$ represent the vector of index $X_{k}$ in $S_{i}$, and $\vec{S}_{l}$ represent the resultant vector of subsystem $S_{i}$. If using index $X_{k}$ to replace subsystem $S_{i}$, the direction loss rate $\lambda_{i}$ of index subsystem $S_{i}$ is: 
$\lambda_{i}=\theta_{k i} / \pi$

$\cos \theta_{k i}=\frac{\overrightarrow{x_{k}} \cdot \overrightarrow{S_{l}}}{\left|\overrightarrow{x_{k}}\right|\left|\overrightarrow{S_{l}}\right|}=\frac{\sum_{t=1}^{m}\left(v_{k t} \sum_{j=1}^{u} v_{j t}\right)}{\sqrt{\sum_{t=1}^{m}\left(v_{k t}\right)^{2}} \sqrt{\sum_{t=1}^{m}\left(\sum_{j=1}^{u} v_{j t}\right)^{2}}}$

where $\theta_{k i} \in[0, \pi]$ is the direction loss degree of subsystem $S_{i}$ when replaced by index $X_{k}$.

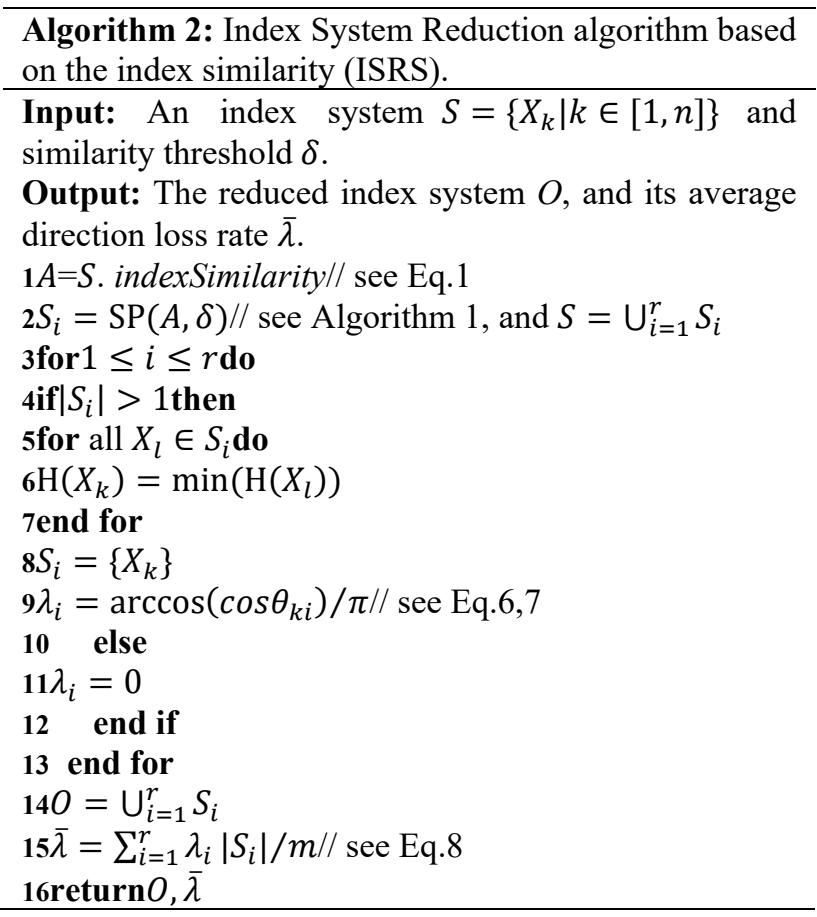

Since there is no similarity between different index subsystems, the evaluation ability loss of the whole index system can be measured through calculating the weighted average of the direction loss rate from each subsystem.

Definition 2 (System Average Direction Loss Rate). Let $S_{i}(i \in[1, r])$ represent the subsystems of index system $S$, The average direction loss rate $\bar{\lambda}$ of index system $S$ is:

$\bar{\lambda}=\sum_{i=1}^{r} \lambda_{i}\left|S_{i}\right| /|S|$

where $\left|S_{i}\right|$ represents the index quantity of subsystem $S_{i}$, and $|S|$ represents the index number of system $S$.

Finally, we propose the index system reduction algorithm based on the index similarity (ISRS), and the pseudo code is shown in Algorithm 2. The time complexity of the ISRS is $O\left(\left(n^{2}+n m\right) / 2\right)$, where $n$ is the total number of indexes and $m$ is the number of samples.

\section{EXPERIMENT AND RESULTS ANALYSIS}

\subsection{Comparison Experiment of Different Algorithms}

In order to verify the effectiveness of the proposed ISRS, we used random number generation to create the dataset with eight indexes and fourteen samples (see Tab.3). Since we limited the value range, all the indexes are under the same scale. Therefore, no further data normalization should be taken.

Comparison experiments are implemented in this section, including different index system reduction methods, i.e., ISRS and PCA (see Section 2.1), as well as different similarity evaluation indices, i.e., Cosine distance and Euclidean distance (see Section 2.2). All the experiments were coded in Matlab 7.8 and run on a personal computer with Windows 7.

Table $3 \mathrm{An}$ index system $S$ with 8 indexes and 14 samples

\begin{tabular}{|c|c|c|c|c|c|c|c|c|}
\hline$O$ & $\mathrm{X}_{1}$ & $\mathrm{X}_{2}$ & $\mathrm{X}_{3}$ & $\mathrm{X}_{4}$ & $\mathrm{X}_{5}$ & $\mathrm{X}_{6}$ & $\mathrm{X}_{7}$ & $\mathrm{X}_{8}$ \\
\hline $\mathrm{O}_{1}$ & 40.4 & 37.05 & 7.2 & 6.1 & 8.3 & 8.7 & 2.442 & 20 \\
\hline $\mathrm{O}_{2}$ & 25 & 19.05 & 11.2 & 11 & 12.9 & 20.2 & 3.542 & 9.1 \\
\hline $\mathrm{O}_{3}$ & 13.2 & 4.95 & 3.9 & 4.3 & 4.4 & 5.5 & 0.578 & 3.6 \\
\hline $\mathrm{O}_{4}$ & 22.3 & 10.05 & 5.6 & 3.7 & 6 & 7.4 & 0.716 & 7.3 \\
\hline $\mathrm{O}_{5}$ & 34.3 & 17.7 & 7.1 & 7.1 & 8 & 8.9 & 1.726 & 27.5 \\
\hline $\mathrm{O}_{6}$ & 35.6 & 18.75 & 16.4 & 16.7 & 22.8 & 29.3 & 3.017 & 26.6 \\
\hline $\mathrm{O}_{7}$ & 22 & 11.7 & 9.9 & 10.2 & 12.6 & 17.6 & 0.847 & 10.6 \\
\hline $\mathrm{O}_{8}$ & 48.4 & 20.1 & 10.9 & 9.9 & 10.9 & 13.9 & 1.772 & 17.8 \\
\hline $\mathrm{O}_{9}$ & 40.6 & 25.65 & 19.8 & 19 & 29.7 & 39.6 & 2.449 & 35.8 \\
\hline $\mathrm{O}_{10}$ & 24.8 & 12 & 9.8 & 8.9 & 11.9 & 16.2 & 0.789 & 13.7 \\
\hline $\mathrm{O}_{11}$ & 12.5 & 14.55 & 4.2 & 4.2 & 4.6 & 6.5 & 0.874 & 3.9 \\
\hline $\mathrm{O}_{12}$ & 1.8 & 0.9 & 0.7 & 0.7 & 0.8 & 1.1 & 0.056 & 1 \\
\hline $\mathrm{O}_{13}$ & 32.3 & 20.85 & 9.4 & 8.3 & 9.8 & 13.3 & 2.126 & 17.1 \\
\hline $\mathrm{O}_{14}$ & 38.5 & 13.65 & 11.3 & 9.5 & 12.23 & 16.4 & 1.327 & 11.6 \\
\hline
\end{tabular}

Table 4 The sample value of the new reduced index system $O^{P C A}$

\begin{tabular}{|c|c|c|c|c|c|c|c|c|}
\hline$O$ & $\mathbf{Z}_{1}$ & $\mathbf{Z}_{2}$ & $\mathrm{Z}_{3}$ & $\mathrm{Z}_{4}$ & $\mathrm{Z}_{5}$ & $\mathrm{Z}_{6}$ & $\mathrm{Z}_{7}$ & $\mathrm{Z}_{8}$ \\
\hline $\mathrm{O}_{1}$ & 12.0 & -20.8 & 9.0 & -3.7 & -0.6 & 0.3 & 0.3 & 0.0 \\
\hline $\mathrm{O}_{2}$ & 0.7 & 5.2 & 1.9 & -7.9 & 1.5 & -0.9 & 0.1 & -0.2 \\
\hline $\mathrm{O}_{3}$ & -25.0 & 3.2 & -1.7 & 1.0 & 0.2 & 0.5 & 0.3 & -0.2 \\
\hline $\mathrm{O}_{4}$ & -15.0 & -1.9 & -2.7 & 0.4 & -1.3 & -0.6 & 0.4 & 0.2 \\
\hline $\mathrm{O}_{5}$ & 5.7 & -9.1 & 1.4 & 11.6 & 0.9 & -0.3 & -0.2 & -0.1 \\
\hline $\mathrm{O}_{6}$ & 23.8 & 10.5 & 0.0 & 1.5 & 1.0 & 0.7 & 0.7 & 0.3 \\
\hline $\mathrm{O}_{7}$ & -4.5 & 7.9 & -0.9 & -2.0 & 0.1 & 0.7 & -0.5 & -0.1 \\
\hline $\mathrm{O}_{8}$ & 14.5 & -12.3 & -9.2 & -0.8 & 0.2 & 0.2 & -0.1 & -0.3 \\
\hline $\mathrm{O}_{9}$ & 40.7 & 14.4 & 4.3 & 1.8 & -1.3 & -0.3 & -0.1 & -0.2 \\
\hline $\mathrm{O}_{10}$ & -2.5 & 4.9 & -1.4 & 0.8 & -0.4 & 0.1 & -0.5 & 0.3 \\
\hline $\mathrm{O}_{11}$ & -21.6 & 0.1 & 5.6 & -3.3 & 0.0 & 0.4 & -0.3 & 0.1 \\
\hline $\mathrm{O}_{12}$ & -38.4 & 5.8 & 2.1 & 3.8 & -0.4 & -0.3 & 0.2 & -0.1 \\
\hline $\mathrm{O}_{13}$ & 4.1 & -5.5 & 1.3 & 0.0 & 0.6 & -0.4 & -0.3 & 0.4 \\
\hline $\mathrm{O}_{14}$ & 5.5 & -2.4 & -9.6 & -3.1 & -0.6 & -0.1 & 0.1 & 0.1 \\
\hline
\end{tabular}

Table 5 Reduction process of ISRS on Cosine and Euclidean distance

\begin{tabular}{|c|c|c|c|}
\hline Indices & Index Subsystems & $O^{\text {ISRS }}$ & $\bar{\lambda}$ \\
\hline & $\left\{\mathrm{X}_{1}, \mathrm{X}_{2}\right\}$ & $\left\{\mathrm{X}_{1}\right\}$ & \\
Cosine & $\left\{\mathrm{X}_{3}, \mathrm{X}_{4}, \mathrm{X}_{5}, \mathrm{X}_{6}\right\}$ & $\left\{\mathrm{X}_{5}\right\}$ & 0.0183 \\
Distance & $\left\{\mathrm{X}_{7}\right\}$ & $\left\{\mathrm{X}_{7}\right\}$ & \\
& $\left\{\mathrm{X}_{8}\right\}$ & $\left\{\mathrm{X}_{8}\right\}$ & \\
\hline \multirow{3}{*}{ Euclidean } & $\left\{\mathrm{X}_{1}\right\}$ & $\left\{\mathrm{X}_{1}\right\}$ & \\
Distance & $\left\{\mathrm{X}_{2}\right\}$ & $\left\{\mathrm{X}_{2}\right\}$ & \multirow{2}{*}{0.0193} \\
& $\left\{\mathrm{X}_{3}, \mathrm{X}_{4}, \mathrm{X}_{5}, \mathrm{X}_{6}, \mathrm{X}_{8}\right\}$ & $\left\{\mathrm{X}_{8}\right\}$ & \\
& $\left\{\mathrm{X}_{7}\right\}$ & $\left\{\mathrm{X}_{7}\right\}$ & \\
\hline
\end{tabular}

Results are shown in Tab. 4 and 5. The comparison experiment results on different index reduction algorithms illustrate that $(1)$ the reduction rate of the PCA $(6 / 8=0.75)$ is larger than the ISRS $(4 / 8=0.5)$. Hence, the index reduction effect of PCA is stronger than the ISRS; (2) the index meaning of the reduced index system $O^{P C A}$ is much more complicated than $O^{I S R S}$, besides, $O^{P C A}$ contains negative value while both the initial index system $\mathrm{S}$ and $O^{I S R S}$ do not exist. Therefore, the results of ISRS is more accessible and available in practice than the PCA.

The comparison experiment results on different similarity evaluation indices illustrate that (1) the direction loss rate of Cosine distance $\left(\overline{\lambda_{\text {cos }}}=0.0183\right)$ is smaller than Euclidean distance $\left(\overline{\lambda_{e u c}}=0.0193\right)$. Hence, as for the ISRS, Cosine distance is more accurate and effective than Euclidean distance. 


\subsection{Index System Reduction of Real Financial Dataset}

After demonstrating the accuracy, this section further verified the stability and efficiency of the ISRS on a real financial dataset with three-hundred enterprise samples. The data structure of this financial dataset in 2015 Resset database is shown in Tab. 6. In the beginning, we conducted the data preprocessing. We identified that the No. 70-90 indexes of three-hundred enterprises are all null value attributes, which are not able to distinguish or evaluate samples. Thus, we removed these indexes from the initial index system $\tilde{S}$, and the formal index reduction experiment only focused on the No. 1-69 indicators.

Fig. 3 describes the result of index subsystems divided by the ISRS, where blue and orange represent two different subsystems respectively, and grey means every index is a subsystem. What's more, the histogram also shows the information entropy of indexes. The ISRS replaced the subsystem in blue with index No. 36, and replaced the subsystem in orange with index No. 64. Finally, we obtained the reduced financial index system $\tilde{O}$ with only forty-five indexes (see Tab. 7). It can be seen that the ISRS achieved great reduction effect on the real dataset, that the reduction rate has already reached 0.5 while keeping the average direction loss rate under 0.023 .

In order to test the stability of the ISRS, we take the reduced index system $\tilde{O}$ as the standard result, and gradually cut down the data size from three-hundred to eighty. Fig. 4 shows the error rate of the ISRS under the decrease of samples. It can be seen that the tendency of this broken line presents three stages, that is the rapid descent stage (80-160), steady descent stage (160-220) and stable fluctuation stage (220-280). For the first stage, the error rate stays in a relatively high level due to too few samples involved in the ISRS calculation. For the second stage, the ISRS has already identified the potential characteristic among these enterprises, and the effect of increasing samples is not as significant as before. For the third stage, the error rate stabilizes at a very low level (0.047), which means the ISRS has converged and its index system reduction result is reliable.
Table 6 The real financial index system $\tilde{S}$ of balance sheets with 90 indexes

\begin{tabular}{|c|l|c|l|c|l|}
\hline No. & \multicolumn{1}{c|}{ Index } & No. & \multicolumn{1}{c|}{ Index } & No. & \multicolumn{1}{c|}{ Index } \\
\hline 1 & Monefd & 31 & TotNcurass & 61 & Surres \\
\hline 2 & Trafinass & 32 & Totass & 62 & Retear \\
\hline 3 & Noterecv & 33 & STloan & 63 & OrdRiskResFd \\
\hline 4 & Accrecv & 34 & Trafindb & 64 & SHEwiomin \\
\hline 5 & Advpay & 35 & Notepay & 65 & minSHE \\
\hline 6 & Intrecv & 36 & Accpay & 66 & SEAdjItems \\
\hline 7 & Othrecv & 37 & Advrecp & 67 & TotSHE \\
\hline 8 & Invtr & 38 & Empsalpay & 68 & LEAdjItems \\
\hline 9 & Defchr & 39 & Taxexppay & 69 & TotliaSHE \\
\hline 10 & Ncurass1Y & 40 & Intpay & 70 & Divrecv \\
\hline 11 & Othcurass & 41 & Divpay & 71 & Consbioass \\
\hline 12 & CAExcItems & 42 & Othaccpay & 72 & Oilgasass \\
\hline 13 & CAAdjItems & 43 & Accrexp & 73 & NCAExcItems \\
\hline 14 & Totcurass & 44 & Ncurlia1Y & 74 & NCAAdjItems \\
\hline 15 & Soldfinass & 45 & Othcurlia & 75 & AssExcItem \\
\hline 16 & Holdinvterm & 46 & Totcurlia & 76 & AssAdjItem \\
\hline 17 & LTrecv & 47 & LTloan & 77 & ImpLoan \\
\hline 18 & LTequinv & 48 & Bdpay & 78 & STbdpay \\
\hline 19 & Invrealest & 49 & LTpay & 79 & Defprcd \\
\hline 20 & Fixass & 50 & Spepay & 80 & Specurlia \\
\hline 21 & Constrpro & 51 & Estmlia & 81 & CLAdjItems \\
\hline 22 & Constrmat & 52 & Deftaxdb & 82 & LExcItem \\
\hline 23 & Dispofixass & 53 & OthNcurlia & 83 & LAdjItems \\
\hline 24 & Prodbioass & 54 & SpeNcurlia & 84 & Treastk \\
\hline 25 & Intanass & 55 & NCLAdjItems & 85 & Spdtrafogcursta \\
\hline 26 & Devlpexp & 56 & TotNcurlia & 86 & Uncfinvlos \\
\hline 27 & Goodwill & 57 & Totlia & 87 & Othres \\
\hline 28 & LTdefchr & 58 & Shrcap & 88 & SEExcItems \\
\hline 29 & Deftaxass & 59 & Capsur & 89 & SEOthEff \\
\hline 30 & OthNcurass & 60 & SpeRes & 90 & LEExcItems \\
\hline & & & & & \\
\hline
\end{tabular}

Table 7 The reduced financial index system $\tilde{O}$ of balance sheet with 45 indexes

\begin{tabular}{|c|l|c|l|c|l|}
\hline No. & \multicolumn{1}{c|}{ Index } & No. & \multicolumn{1}{c|}{ Index } & No. & \multicolumn{1}{c|}{ Index } \\
\hline 2 & Trafinass & 26 & Devlpexp & 47 & LTloan \\
\hline 3 & Noterecv & 27 & Goodwill & 49 & LTpay \\
\hline 5 & Advpay & 28 & LTdefchr & 50 & Spepay \\
\hline 6 & Intrecv & 29 & Deftaxass & 51 & Estmlia \\
\hline 7 & Othrecv & 30 & OthNcurass & 52 & Deftaxdb \\
\hline 8 & Invtr & 35 & Notepay & 53 & OthNcurlia \\
\hline 10 & Ncurass1Y & 36 & Accpay & 54 & SpeNcurlia \\
\hline 11 & Othcurass & 37 & Advrecp & 56 & TotNcurlia \\
\hline 15 & Soldfinass & 38 & Empsalpay & 58 & Shrcap \\
\hline 18 & LTequinv & 39 & Taxexppay & 59 & Capsur \\
\hline 19 & Invrealest & 40 & Intpay & 60 & SpeRes \\
\hline 21 & Constrpro & 41 & Divpay & 61 & Surres \\
\hline 22 & Constrmat & 42 & Othaccpay & 62 & Retear \\
\hline 24 & Prodbioass & 44 & Ncurlia1Y & 64 & SHEwiomin \\
\hline 25 & Intanass & 45 & Othcurlia & 65 & minSHE \\
\hline
\end{tabular}

\section{INDEX SYSTEM REDUCTION PROCESS}

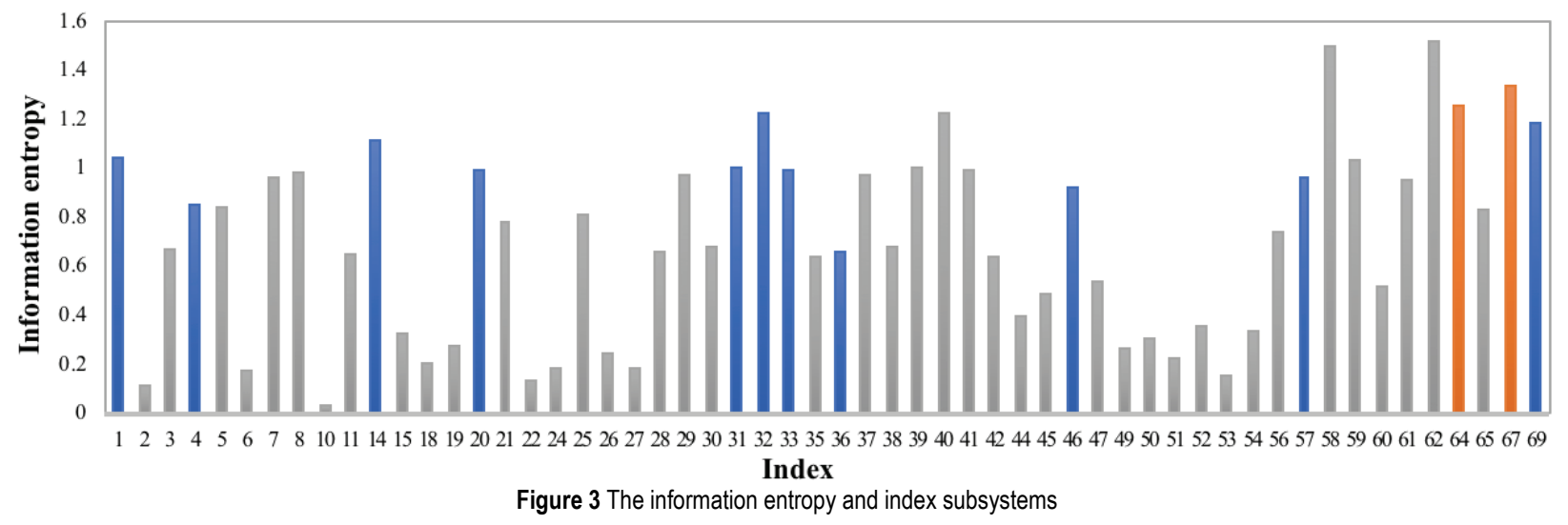

The experimental results on the real dataset illustrates that the ISRS is more applicable to the large-sample dataset. 
STABILITY ANALYSIS

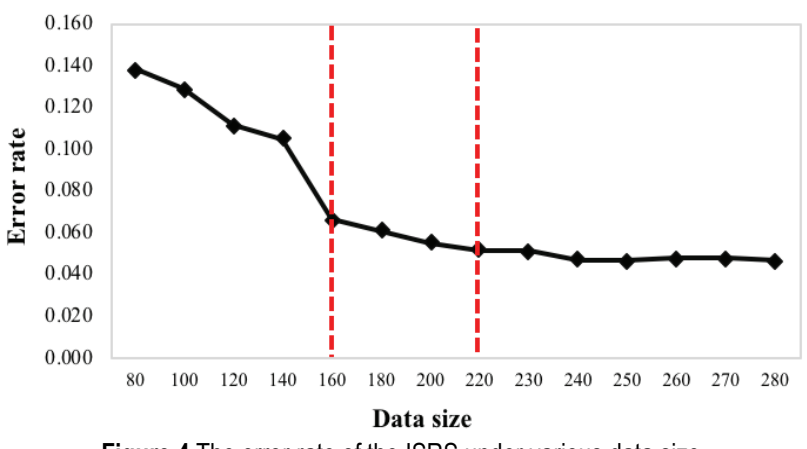

Figure 4 The error rate of the ISRS under various data size.

\section{CONCLUSIONS}

Index system reduction has become one of the most popular research fields since its first appearance, and is widely applied to the multi-attribute decision making (MADM). Unlike the previous approaches (like PCA) that replace indexes with new components, this paper studies the index system reduction problem from the perspective of system structure partition.

We proposed and demonstrated the Index Subsystem Judgement theorem, that improves the efficiency of the classic system structure partition algorithm, i.e., the interpretative structural modeling (ISM). An algorithm of index system reduction based on the index similarity (ISRS) was also proposed. The ISRS is able to reduce the index quantity while still keeping the index meaning, through directly selecting the most representative index from each index subsystem. Moreover, the average direction loss rate was put forward, which successfully measures the evaluation ability loss of the index system during reduction.

Experiments on both synthetic and real-world datasets illustrate that the ISRS is able to converge under the dataset with sufficient samples, and obtain more accessible and available reduction results in practice.

The future work of our study is to improve the efficiency of ISRS, especially for large-scale data analysis. Also, we will further verify the performance of our proposed method through more practical problems.

\section{Acknowledgements}

The study is supported by national natural science foundation of China (71272161) and China Scholarship Council.

\section{REFERENCES}

[1] Bi, Z., Xu, F., Lei, J., \& Jiang, T. (2016). Attribute reduction in decision-theoretic rough set model based on minimum decision cost. Concurrency and Computation-Practice \& Experience, 28(15), 4125-4143. https://doi.org/10.1002/cpe.3830

[2] Castro, D. M. \& Parreiras, F. S. (2018). A Review on MultiCriteria Decision-Making for Energy Efficiency in Automotive Engineering. Applied Computing and Informatics. In Press. https://doi.org/10.1016/j.aci.2018.04.004

[3] Chen, J., Lin, Y., Lin, G., Li, \& Y. Zhang, Y. (2017). Knowledge-Based Systems Attribute reduction of covering decision systems by hypergraph model. Knowl. Based Syst., 118(1), 93-104. https://doi.org/10.1016/j.knosys.2016.11.010
[4] Dubey, A. K. \& Yadava, V. (2008). Multi-objective optimization of Nd: YAG laser cutting of nickel-based superalloy sheet using orthogonal array with principal component analysis. Opt. Lasers Eng., 46(2), 124-132. https://doi.org/10.1016/j.optlaseng.2007.08.011

[5] Han, J. (2012). Data Mining: Concepts and Techniques, third ed., China Mach. Press, Beijing.

[6] Huang, J., Zhou, Z., Gao, Z., Zhang, M., \& Yu, L. (2017). Aerodynamic multi-objective integrated optimization based on principal component analysis. Chinese J. Aeronaut., 30(4), 1336-1348. https://doi.org/10.1016/j.cja.2017.05.003

[7] Jeya, G., Sekar, V., \& Vimal, K. (2016). Application of interpretative structural modelling integrated multi criteria decision-making methods for sustainable supplier selection. J. Model. Manag., 11(2), 358-388. https://doi.org/10.1108/JM2-02-2014-0012

[8] Kamble, P. D., Waghmare, A. C., Askhedkar, R. D., \& Sahare, S. B. (2017). Multi objective optimization of turning parameters considering spindle vibration by Hybrid Taguchi Principal Component Analysis (HTPCA). Mater. Today: Proceedings, 4(2), 2077-2084. https://doi.org/10.1016/j.matpr.2017.02.053

[9] Kapsoulis, D., Tsiakas, K., Trompoukis, X., Asouti,V., \& Giannakoglou, K. (2018). Evolutionary multi-objective optimization assisted by metamodels, kernel PCA and multicriteria decision making techniques with application in aerodynamics. Appl. Soft Comput., 64(1), 1-13. https://doi.org/10.1016/j.asoc.2017.11.046

[10] Li, H., Li, D., Zhai, Y., Wang, S., \& Zhang, J. (2016). A novel attribute reduction approach for multi-label data based on rough set theory. Inf. Sci. (Ny)., 367-368(1), 827-847. https://doi.org/10.1016/j.ins.2016.07.008

[11] Li, F., Yang, J., Jin, C., \& Guo, C. (2017). A new effectbased roughness measure for attribute reduction in information system. Inf. Sci. (Ny)., 378(1), 348-362. https://doi.org/10.1016/j.ins.2016.08.056

[12] Lin, Y., Li, Y., Wang, C., \& Chen, J. (2018). Attribute reduction for multi-label learning with fuzzy rough set. Knowledge-Based Systems, 152(15), 51-61. https://doi.org/10.1016/j.knosys.2018.04.004

[13] Li, J. \&Wang, X. (2016).Decision reduction for object oriented concept lattices.Computer Engineering and Applications. 52 (18), 154-157. https://doi.org/10.3778/j.issn.1002-8331.1512-0353

[14] Ma, W. \& Mao, G. (2009). System structure partition based on graph theory and matrix theory. J. Lanzhou Jiaotong Univ., 6(1), 150-154.

[15] Niculescu, M., Irimia, C., Rosca, L., Grovu, M., \& Guiman, M. (2017). Structural dynamic applications using principal component analysis. CONAT 2016, 90-99. https://doi.org/10.1007/978-3-319-45447-4_10

[16] Pozo, C., Ruiz-Femenia, R., Caballero, J., Guillen-Gosalbez, G., \& Jimenez, L. (2012). On the use of Principal Component Analysis for reducing the number of environmental objectives in multi-objective optimization: Application to the design of chemical supply chains. Chem. Eng. Sci., 69(1), 146-158. https://doi.org/10.1016/j.ces.2011.10.018

[17] Qi, M., Fu, Z., Jing, Y., \& Ma, Y. (2013). A Comprehensive Evaluation Method of Power Plant Units Based on Information Entropy and Principal Component Analysis. Proceedings CSEE, 33(2), 58-65. https://doi.org/10.13334/j.0258-8013.pcsee.2013.02.015

[18] Raul, P. J., Catherine, A.-P., \& Stephan, A. (2018). Combining multi-objective optimization, principal component analysis and multiple criteria decision making for ecodesign of photovoltaic grid-connected systems. Sustain. Energy Technol. Assessments, 27(1), 94-101. https://doi.org/10.1016/j.seta.2018.03.008 
[19] Sun, L. \& Qian, W. (2009). Research on the comprehensive evaluation method based on principal component analysis. Math. Pract. Theory., 18(1), 15-20.

[20] Singarvel, B., Selvaraj, T., \& Jeyapaul, R. (2014). Multi objective optimization in turning of EN25 Steel Using Taguchi Based Utility Concept Coupled with Principal Component Analysis. Procedia Eng., 97(1), 158-165. https://doi.org/10.1016/j.proeng.2014.12.237

[21] Sabio, N., Kostin, A., Guillen-Gosalbez, G., \& Jimenez, L. (2012). Holistic minimization of the life cycle environmental impact of hydrogen infrastructures using multi-objective optimization and principal component analysis. Int. J. Hydrogen Energy., 37(6), 5385-5404. https://doi.org/10.1016/j.jijhydene.2011.09.039

[22] Sinha, P., Kumar, R., Singh, G. K., \& Thomas, D. (2015). Multi-Objective Optimization of Wire EDM of AISI D3 Tool Steel Using Orthogonal Array with Principal Component Analysis. Mater. Today Proc., 2(1), 3778-3787. https://doi.org/10.1016/j.matpr.2015.07.183

[23] Vluymans, Ys., Cornelis, C., Herrera, F., \& Saeys, Y. (2018). Multi-label classification using a fuzzy rough neighborhood consensus. Inf. Sci. (Ny)., 433-434(1), 96-114. https://doi.org/10.1016/j.ins.2017.12.034

[24] Wu, S., Gao, X., \& B. M. (2003). Data Warehousing and Data Mining, first ed., Metallurgical Industry Press, Beijing.

[25] Wang, Z., Liu, J., Zhang, Q., Jiang, Y. (2015). Attribute reduction of the index system in circular economy based on rough sets. Stat. Decis., 6(1) 34-38.

[26] Wonggasem, K., Wagner, T., Trautmann, H., Biermann, D., \& Weihs, C. (2013). Multi-objective Optimization of Hard Turning of AISI 6150 Using PCA-based Desirability Index for Correlated Objectives. Procedia CIRP, 12(1), 13-18. https://doi.org/10.1016/j.procir.2013.09.004

[27] Yi, S., Lai, Z., He, Z., Cheung, Y., \& Liu, Y. (2017). Joint sparse principle component analysis. Pattern Recognit., 61(1), 524-536. https://doi.org/10.1016/j.patcog.2016.08.025

[28] Yu, Y., Pedrycz, W., \& Miao, D. (2013). Neighborhood rough sets based multi-label classification for automatic image annotation. Int. J. Approx. Reason., 54(1), 1373-1387. https://doi.org/10.1016/j.jiar.2013.06.003

[29] Yu, Y., Miao, D., Zhang, Z., \& Wang, L. (2013). Multi-label Classification Using Rough Sets. Lect. Notes Artif. Intell., 119-126. https://doi.org/10.1007/978-3-642-41218-9_13

[30] Yu, Y., Pedrycz, W., Miao, D. \& Zhang, H. (2013). Neighborhood Rough Sets Based Multi-label Classification. Proc. 2013 Jt. IFSA World Congr. Nafips. Annu. Meet., 8690. https://doi.org/10.1109/IFSA-NAFIPS.2013.6608380

[31] Yu, Y., Miao, D., Zhao, C., \& Wang, Y. (2015). Knowledge Acquisition Methods for Multi-Label Decision System Based on Rough Sets. J. Front. Comput. Sci. Technol., 9(1), 94-104. https://doi.org/10.3778/j.issn.1673-9418.1406024

[32] Zhu, L. \& Lv, B. (2004). Simple and convenient method of ISM. Syst. Eng. Electron., 12(1), 1815-1817.

[33] Zhang, Z., Zhao, T., \& Chunhong, W. (2009). Application of the attribute reduction method based on rough set. Sci. Technol. Manag. Res., 1(1), 78-85.

[34] Zhang, L., Hu, Q., Zhou, Y., \& Wang, X. (2014). Multi-label Attribute Evaluation Based on Fuzzy Rough Sets. Lect. Notes Comput. Sci., 8536(1), 100-108. https://doi.org/10.1007/978-3-319-08644-6_10

[35] Chatterjee, K., Pamucar, D., \& Zavadskas, E. K. (2018). Evaluating the performance of suppliers based on using the R'AMATEL-MAIRCA method for green supply chain implementation in electronics industry. Journal of Cleaner Production, 184(1), 101-129. https://doi.org/10.1016/j.jclepro.2018.02.186

[36] Jagannath, R., Krishnedu, A., Samarji, K., \& Dragan, P. (2018). A rough strength relational DEMATEL model for analysing the key success factors of hospital service quality.
Decision Making: Applications in Management and Engineering, 1(1), 121-142. https://doi.org/10.31181/dmame1801121r

[37] Pamucar, D., Chatterjee, K., \& Kazimieras Zavadskas, E. (2019).Assessment of third-party logistics provider using multi-criteria decision-making approach based on interval rough numbers. Computers and Industrial Engineering, 127(1), 383-407. https://doi.org/10.1016/j.cie.2018.10.023

[38] Stević, Ž., Đalić, I., Pamučar, D., Nunić, Z., Vesković, S., Vasiljević, M., \& Tanackov, I. (2019). A new hybrid model for quality assessment of scientific conferences based on rough BWM and SERVQUAL. Scientometrics, 119(1), 1-30. https://doi.org/10.1007/s11192-019-03032-z

[39] Karavidic, Z. \& Projovic, D. (2018). A multi-criteria decision-making (MCDM) model in the security forces operations based on rough sets. Decision Making: Applications in Management and Engineering, 1(1), 97-120. https://doi.org/10.31181/dmame180197k

\section{Contact information}

\section{Ai WANG, PhD student}

(Corresponding author)

University of Science and Technology Beijing,

No. 30 Xueyuan Road, Haidian District, Beijing, China

wangai22222@126.com

\section{Xuedong GAO, Professor}

University of Science and Technology Beijing,

No. 30 Xueyuan Road, Haidian District, Beijing, China

gaoxuedong@manage.ustb.edu.cn 